\title{
Kinematic, Workspace and Static Analysis of a Upper Body Humanoid Robot
}

\author{
Shifa Sulaiman, A P Sudheer
}

\begin{abstract}
Humanoid robots are used fortraining purposes, personal assistance, understanding the human body structure and behavior, health care field, entertainment field, military purposes, space explorations, etc. Kinematic analysis plays a crucial role in the development of a humanoid robot. This paper presents the kinematic, workspace and static analysis of a Humanoid upper body robot. The forward kinematic model is obtained by using Screw theory. Screw theory provides the complete description of the system than the Denavit-Hartenberg (DH) method. Screw theory decreases the chances of occurrences of singularities inside the workspace. The joint angles in the upper body are obtained by using cubic spline trajectory method. The proposed torso and arm design can imitate the human body postures. The humanoid robot is designed with 3 Dofs in the torso, 2 Dofs in the neck and 5 Dofs in each arm. The two arms are designed with identical joints.
\end{abstract}

Index Terms - Humanoid Robot, Kinematic analysis, workspace, static analysis.

\section{INTRODUCTION}

Humanoid robots are used widely in various fields. The researches associated with humanoid robotics are also increasing day by day. In most of the humanoid robots, the degree of freedom of the torso is limited to one or two. Humanoid upper body robots are exhibiting very low payload capacity. Most of the upper body robots are employed with wheeled platform than legged .Upper body motions are not offering stability at high walking or running speeds. Humanoid upper body robots lacks good control design strategies for imitating human like gait patterns. Energy consumption at various joints are found to be high. Chances of upper body collapse and self collision during locomotion is also very high.

Kinematic analysis of a humanoid robot based on screw theory is discussed in Man et al. [1]. Comparison of screw theory with other methods is also done. Doria et al. [2] introduced amethod to solve inverse kinematics of a robotic system by using spline functions. Three variate splines are used as the functions for solving inverse kinematic solutions.Xinget al. [3] explained a methodto compensate for the yaw movement of a humanoid robot during walking. The trunk spin motion and the arms swinging motion are proposed to compensate for the yaw moment during walk ing. This paper analyzed the moment generated during the locomotion of the humanoid robot. ZMP is taken as the stability criteria. The DOF of the waist is constrained to one. Constant height of centre of mass is maintained during the walking cycle. Straight Knee walking pattern is not achieved by using this method. Petar et al. [4] depicted a method to control the upper and lower body of the robot by using

Revised Manuscript Received on April 12, 2019.

ShifaSulaiman, Research Scholar, NIT, Calicut, Kerala, India.

Dr. A P Sudheer, Assistant Professor, NIT, Calicut, Kerala, India. different control strategies. The hip based balance controller allowed the robot to increase the size of the workspace. The proposed method is based on two main techniques such as imitation learning and disturbance rejection with reaction null space method. In this work, the generalization abilities of the position and force encoding technique are not fully tested. Fukayaet al. [5] introduced the design of a new humanoid-type hand with human-like manipulation abilities. The new hand is designed for the humanoid robot ARMAR Ultrasonic motors are used as actuators. Sudden changes in grasping styles are not possible by using this method.Kapse et al. [6] introduced a new type of modular design of humanoid robot arm with more flexibility and resembles actual human arm in movement and appearance. Themechanical modular design is small and compact compared to currently available humanoid robot arms. Pneumatic actuators are used for actuating joints. But Pneumatic actuators cannot be used for picking heavy objects. Physical attributes are represented as B-spline explicit functions in Ruchanurucks[7]. The B-spline coefficients are calculated to limit the physical attributes The explicit method used here is better than many other methods that only reduce such physical attributes using objective functions. This method is applicable to both offline and online mode of operation.

Based on literature review, many research gaps were identified. The main goal of this paper is to design a humanoid upper body, which can be placed above wheeled, tracked, legged or hybrid platforms for better stability. This paper focuses on kinematic, workspace and static analysisofa upper body humanoid robot. Due to the presence of higher degrees of freedom, kinematic and dynamic analysis is a difficult task to complete. The upper body of humanoid robot plays an important role for stabilized motion. For imitating human like body movements and gait pattern, the upper body should also be designed in a proper manner.

\section{KINEMATIC ANALYSIS OF UPPER BODY}

The upper body humanoid robots consist of 15 Degrees of freedom. The two arms are composed of 5 Dof each and torso portionconsist of 3 Dofhipjoints and 2 Dof neck joints. Kinematic as well as dynamic analysis of the model by using screw theory is derived. The kinematic model is the analytical description of the spatial geometry of motion of the manipulator with respect to a fixed reference frame. Kinematic equations are the functional relationship between joint parameters, position and orientation of the end effector. Screw theory approach [8] is used for kinematic modeling. 
This method is not applied much in humanoid robotics.

The method uses inertial frames. One of the frames will be assigned to the base and other to the end effector. The idea of representing displacement of rigid bodies based on motion of a screw with a defined pitch is the base for this method.Each joint is assigned with a screw axis with zero pitch for rotary joints. The velocities are represented as $6 \mathrm{x} 1$ vectors called twists. The joint forces are represented as $6 \times 1$ vectors called wrenches.

Rodrigues formula is given by

$\mathrm{R}(\widehat{\omega}, \theta)=\mathrm{e}^{[\widehat{\omega]} \theta}=\mathrm{I}+\sin \theta[\widehat{\omega}]+(1-\cos \theta)\left[\widehat{\omega}^{2}\right.$

Transformation matrix

is givene $^{[\mathrm{S}] \theta}=$

$\left[\begin{array}{cc}\mathrm{e}^{[\widehat{\omega}] \theta} & (\mathrm{I} \theta+(1-\cos \theta)[\widehat{\omega]}] \\ 0 & 1\end{array}+(\theta-\sin \theta)\left[\widehat{\omega}^{2} \mathrm{v}\right]\right.$ Screw axis is given by,

$\mathrm{S}=\left[\begin{array}{l}\omega \\ \mathrm{v}\end{array}\right]$

$\|\omega\|=1$, for revolute joints

$\|\omega\|=0$ for linear joints

Where $\mathrm{g}$ is the axis of screw and $\mathrm{p}$ is the pitch

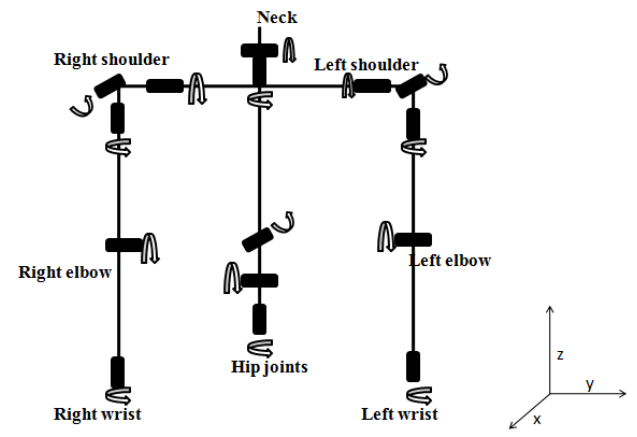

Fig 1. Upper body model

The final transformation matrix of end effector frame with respect to base frame with nlinksis given by

$$
\mathrm{G}(\theta)=\mathrm{e}^{\left[\mathrm{S}_{1}\right] \theta_{1}} \mathrm{e}^{\left[\mathrm{S}_{2}\right] \theta_{2}} \ldots \ldots \mathrm{e}^{\left[\mathrm{S}_{\mathrm{n}}\right] \theta_{\mathrm{n}}} \cdot \mathrm{M}
$$

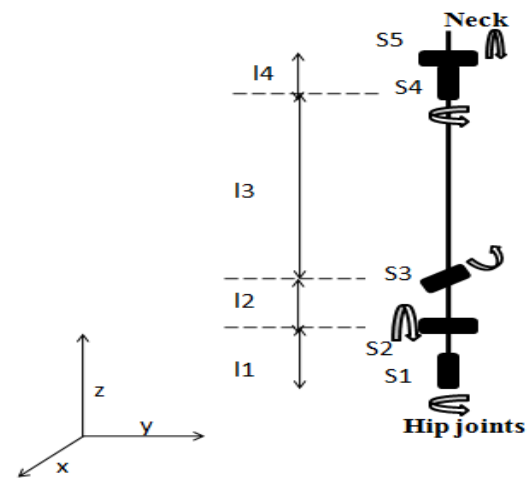

Fig 2. Screw axes of hip joints and neck joints

The transformation of the neck with respect to base frame is given by equation (4)

$$
H_{T_{B}}=e^{s_{1} \theta_{1}} e^{s_{2} \theta_{2}} e^{s_{3} \theta_{3}} e^{s_{4} \theta_{4}} e^{s_{5} \theta_{5}} M
$$

Where, $\mathrm{v}=-\omega \times \mathrm{g}+\mathrm{p} \omega$

$$
\begin{aligned}
e^{s_{1} \theta_{1}} & =\left[\begin{array}{cccc}
c 1 & -s 1 & 0 & 0 \\
s 1 & c 1 & 0 & 0 \\
0 & 0 & 1 & 0 \\
0 & 0 & 0 & 1
\end{array}\right] \\
e^{s_{2} \theta_{2}} & =\left[\begin{array}{cccc}
c 2 & 0 & s 2 & -l 2 s 2 \\
0 & 1 & 0 & 0 \\
-s 2 & 0 & c 2 & c 2-1 \\
0 & 0 & 0 & 1
\end{array}\right] \\
e^{s_{3} \theta_{3}} & =\left[\begin{array}{cccc}
1 & 0 & 0 & 0 \\
0 & c 3 & -s 3 & -(l 2+l 2) * s 3 \\
0 & s 3 & c 3 & -(l 1+l 2) *(1-c 3) \\
0 & 0 & 0 & 1
\end{array}\right] \\
e^{s_{4} \theta_{4}} & =\left[\begin{array}{cccc}
c 4 & -s 4 & 0 & 0 \\
s 4 & c 4 & 0 & 0 \\
0 & 0 & 1 & 0 \\
0 & 0 & 0 & 1
\end{array}\right] \\
e^{s_{5} \theta_{5}} & =\left[\begin{array}{cccc}
c 5 & 0 & s 5 & -(l 1+l 2+l 3+l 4) s 5 \\
0 & 1 & 0 & 0 \\
-s 5 & 0 & c 5 & -(l 1+l 2+l 3+l 4)(c 5-1) \\
0 & 0 & 0 & 1
\end{array}\right] \\
M & =\left[\begin{array}{cccc}
1 & 0 & 0 \\
0 & 0 & 0 & 0 \\
0 & 1 & (l 1+l 2+l 3+l 4) \\
0 & 0 & & 1
\end{array}\right]
\end{aligned}
$$

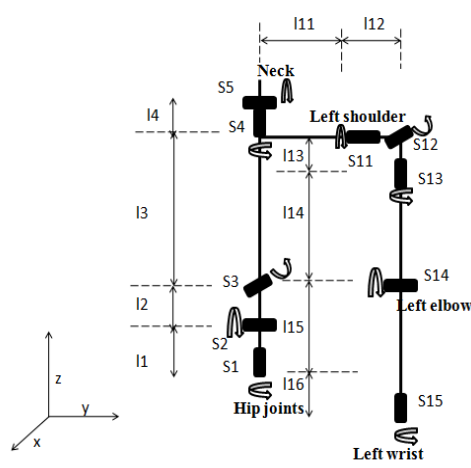

Fig 3. Screw axes of Left arm

Similarly,

The transformation of the left arm end effectorwith respect tofirst hip joint (base frame) is given by equation (4)

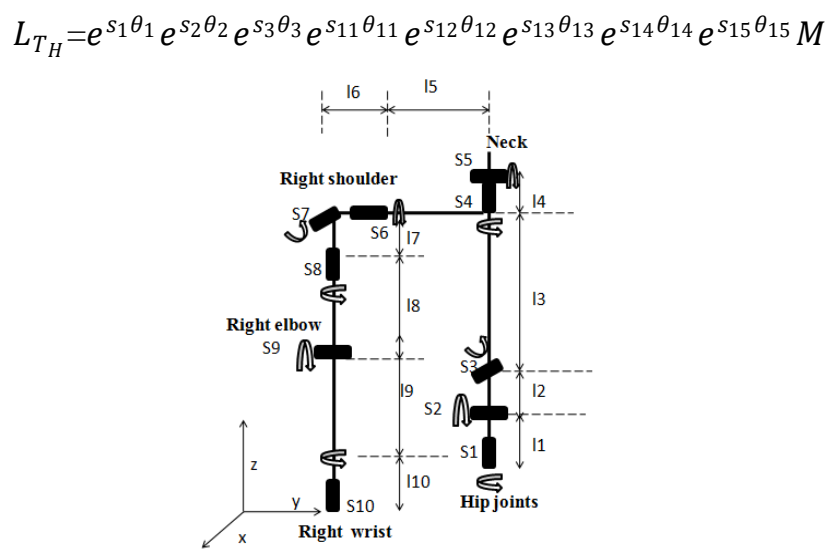

Fig 4. Screw axes of Rightarm

Published By: 
The transformation of the Right arm end effector with respect to first hip joint (base frame) is given by equation (4)

$$
R_{T_{B}}=e^{s_{1} \theta_{1}} e^{s_{2} \theta_{2}} e^{s_{3} \theta_{3}} e^{s_{6} \theta_{6}} e^{s_{7} \theta_{7}} e^{s_{8} \theta_{8}} e^{s_{9} \theta_{9}} e^{s_{10} \theta_{10} M}
$$

\section{WORKSPACE ANALYSIS}

The combined workspace for the upper bodyis obtained as shown in figure 6.This plot of the upper body gives an idea about the required workspace for the coordinated motion, while the end effectors are moving through the cubic trajectory. The workspace volume is also calculated. The obtained workspace is found to be satisfying for the preferred tasks.

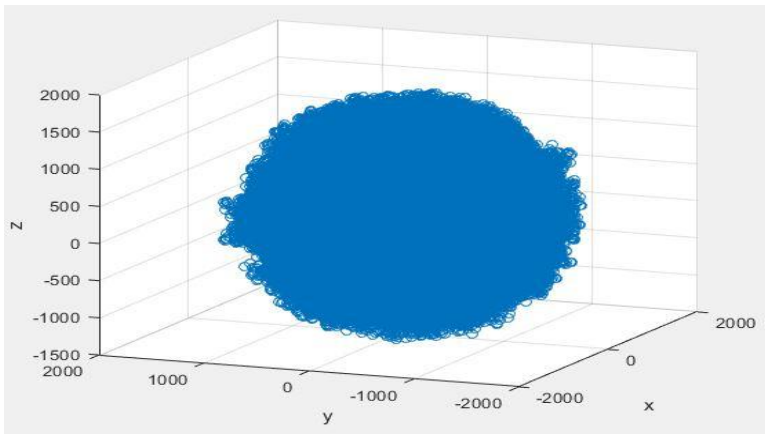

Fig 6. Workspace of upper body

\section{INVERSE KINEMATIC SOLUTIONS}

The Iterative method is used for obtaining inverse kinematic solutions. The inverse solutions for upper body are solved in Matlab by using Levenberg - Marquardt algorithm. The inverse solutions are obtained by assuming cubic spline trajectories through neck joints as well as right and left arm end effector joints. The cubic spline is plotted by inputting coordinate values from workspace. The simulation results of upper body by assuming coordinated motions for neck as well as arm joints are obtained as shown in figure 7.

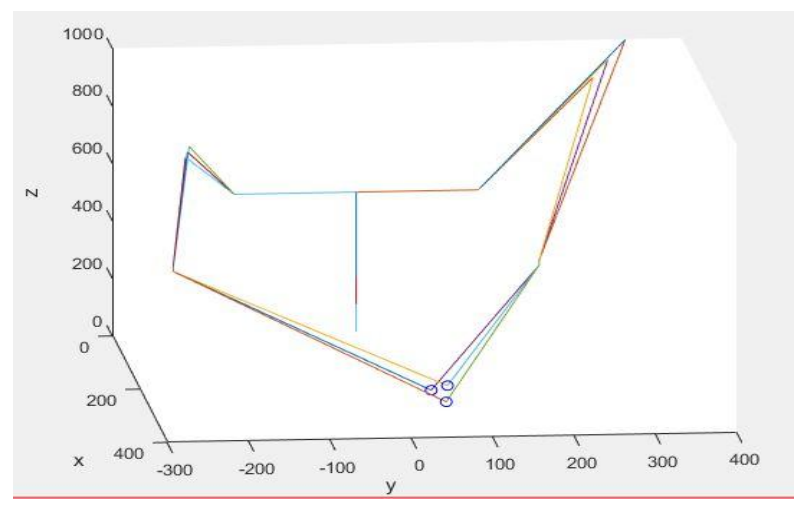

Fig 7. Simulation result of upper body movements

\section{STATIC ANALYSIS\& RESULTS}

Static structural analysis also carried out along with the kinematic analysis for the dimensional synthesis. The Static analysis of the upper body links are carried out in ANSYS software as shown in figure 8 and 9 . Induced stresses and deformations are determined for various links through the analysis. Optimization of links based on varying thickness is also done.Aluminium is selected as the material for upper body. The stresses acting in the upper body are found to be less than the yield strength of Aluminium with a safety factor of3.

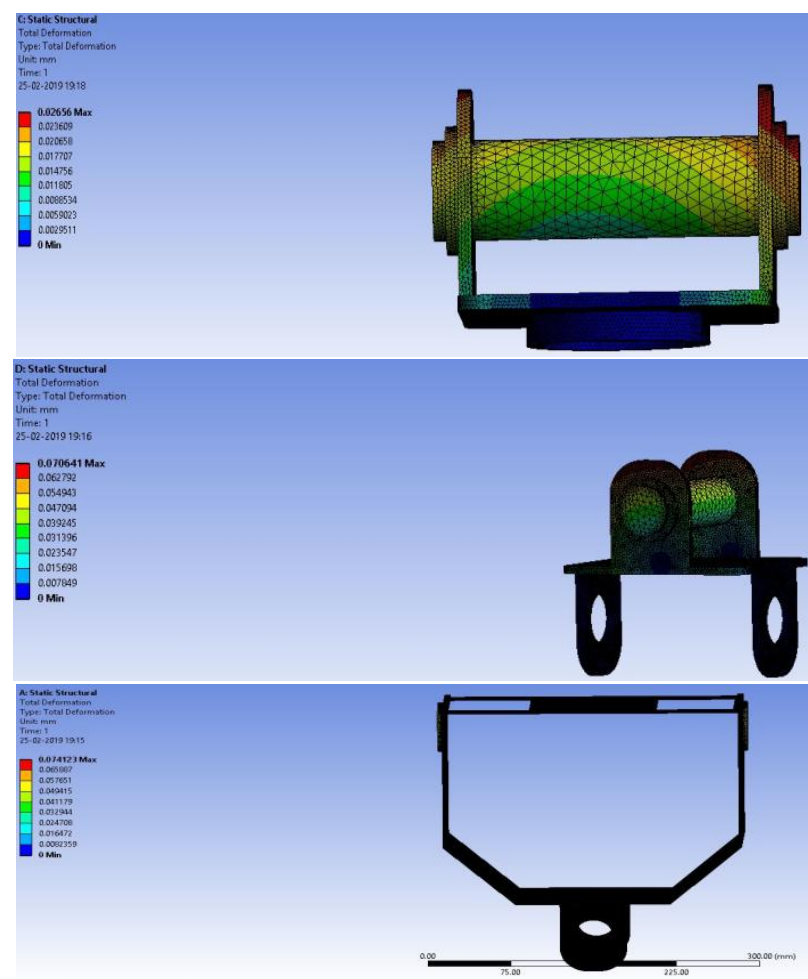

Fig8.Deformations invarious links in upper body

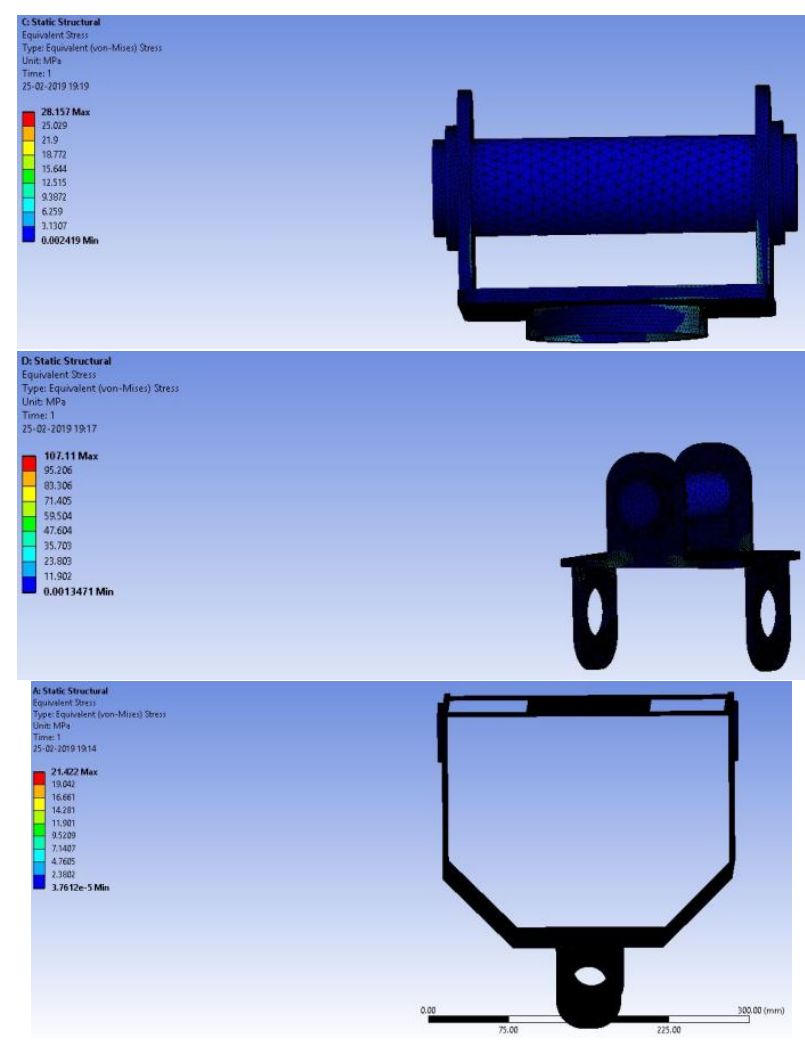

Fig9. Stressesin various links of upper body 


\section{CONCLUSION}

In this paper, kinematic, workspace and static analysis of aupper body humanoid robot is done. Kinematic equations are derived based on screw theory. Inverse kinematic solutions are obtained by using analytical method by assuming a cubic spline through the joints. Workspace analysis is also done. Static analysis is also completed and the designed components are found to bein safe range. Dynamic analysis should be carried out to find out the forces and torques acting on the joints. After completing dynamic analysis, motors should be selected for actuating the joints. The upper body robot developed in this work can be placed above wheeled, legged, tracked or hybrid platforms for future works. Theupper body system developed based on screw theory will be compared with DH modeled systems. optimization and stability analysis of upper body can be done. A good control system design should be adopted for operation of joints.

\section{REFERENCES}

1. Man, C. H., Xun, F., Li, C. R., \& Zhao, Z. H. (2007). Kinematics analysis based on screw theory of a humanoid robot. Journal of China University of Mining and Technology, 17(1), 49-52.

2. Doria, A., Angrili, F., \& De Marchi, S. (1993). Inverse kinematics robot calibration by spline functions. Applied mathematical modelling, 17(9), 492-498.

3. Xing, D., \& Su, J. (2010). Arm/trunk motion generation for humanoid robot. Science China Information Sciences, 53(8), 1603-1612.

4. PetarKormushev, Dragomir N. Nenchev, Sylvain Calinon and Darwin G. Caldwell,(2011) 'Upper-body Kinesthetic Teaching of a Free-standing Humanoid Robot' IEEE International Conference on Robotics and Automation, 3970 3975.

5. Fukaya, N., Toyama, S., Asfour, T., \&Dillmann, R. (2000). Design of the TUAT/Karlsruhe humanoid hand. InProceedings. 2000 IEEE/RSJ International Conference on Intelligent Robots and Systems (IROS2000) (Cat. No. 00CH37113)3,1754-1759.

6. Kapse S.S., Kapse S.K. (2013) 'Modular Design and Control of Humanoid Robot Arm', International Journal of Scientific \& Engineering Research, 4(12), 1390-1395.

7. M. Ruchanurucks, (2015) 'Humanoid robot upper body motion generation using B-spline-based functions' Robotica, 33(4), 705-720.

8. Kevin M Lynch and Frank C Park 2017 Modern RoboticsMechanics, Planning and Control ( $1^{\text {st }}$ edition $)$,Cambridge University press, Chapter 4

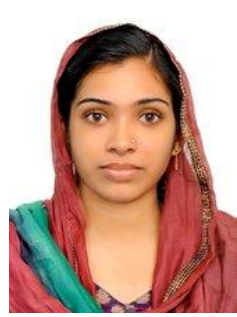

\section{AUTHORS PROFILE}

ShifaSulaimanis a Ph.D. research scholar inMechanical Engineering Department, National Institute of Technology Calicut (NITC). She has 5.5 years of experience in teaching at various educational institutions. She is specialized in Humanoid Robotics.She is currently doing research in upper body humanoid robotics.

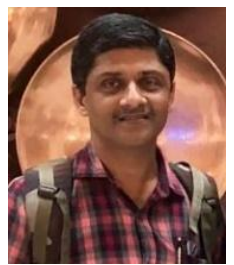

Dr. Sudheer. A. $\mathbf{P}$ is currently working asa Assistant Professor, in the Mechanical Engineering department, National Institute of Technology Calicut (NITC). He is in teaching, research and academic administration for the last 20 years. He has nearly 30 publications in International and national journals and conferences. He is guiding M.Tech and PhDs students. He has filed for more than 5 patents in the area of robotics. His research interests includes kinematics, dynamics and control of robotics, mobile robotics, under water robots and vision systems. He is a member of "the Robotics Society India"(TRS-India) and ISTE. Presently he is the editor of TRS India. 